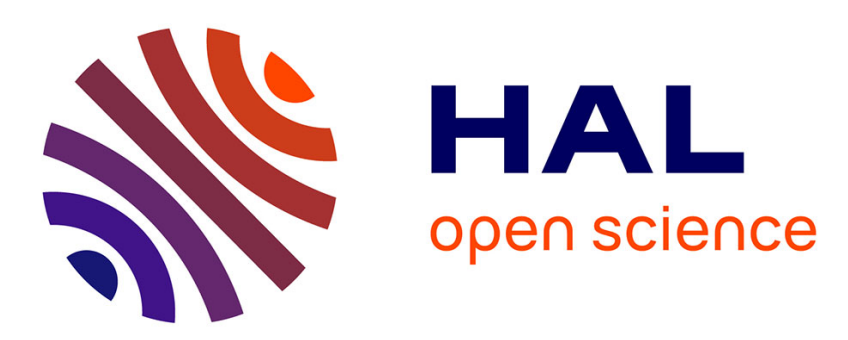

\title{
A novel comanipulation device for assisting needle placement in ultrasound guided prostate biopsies
}

Cécile Poquet, P Mozer, Guillaume Morel, Marie-Aude Vitrani

\section{To cite this version:}

Cécile Poquet, P Mozer, Guillaume Morel, Marie-Aude Vitrani. A novel comanipulation device for assisting needle placement in ultrasound guided prostate biopsies. 2013, 10.1109/IROS.2013.6696941 . hal-01170728

\section{HAL Id: hal-01170728 \\ https://hal.sorbonne-universite.fr/hal-01170728}

Submitted on 2 Jul 2015

HAL is a multi-disciplinary open access archive for the deposit and dissemination of scientific research documents, whether they are published or not. The documents may come from teaching and research institutions in France or abroad, or from public or private research centers.
L'archive ouverte pluridisciplinaire $\mathbf{H A L}$, est destinée au dépôt et à la diffusion de documents scientifiques de niveau recherche, publiés ou non, émanant des établissements d'enseignement et de recherche français ou étrangers, des laboratoires publics ou privés. 


\title{
A novel comanipulation device for assisting needle placement in ultrasound guided prostate biopsies
}

\author{
C. Poquet, P. Mozer, G. Morel, M.-A. Vitrani
}

\begin{abstract}
A novel robotic device, aimed at assisting a urologist in performing prostate biopsies guided by an endorectal ultrasound probe, is described.

The paper describes the robot kinematics and the actuation system. The actuation system combines electromagnetic brakes, balancing springs, and electrical motors with cable transmissions. The robot supports two modes of operation: the free mode, where the entire control of the probe movements is left to the urologist, and the blocked mode, where the robot precisely maintains the probe at a given position and orientation with respect to the prostate.

For the blocked mode, the set of specifications is antagonistic: firstly, a security constraint requires a low robot stiffness to allow to compliantly adapt to potential movements from the patient; secondly, a precision constraint requires a high robot stiffness in order to maintain the position and orientation of the probe in the presence of unmodeled external forces, when the robot is switched from the free mode to the blocked mode. A control strategy is developed to obtain this behavior. It combines an inner impedance controller with a relatively low stiffness and an outer intelligent position integrator that operates only during a limited period of time, when switching from the free mode to the blocked mode.
\end{abstract}

Both in vitro and in cadavero experimental results show the efficiency of this approach.

\section{INTRODUCTION}

Among applications that are widely addressed in the domain of medical robotics, positioning a needle in a patient's prostate has received a particular attention over the past decade. Indeed, this application has a large health impact, while exhibiting a number of technical difficulties for which robotics is expected to provide some help.

Cancerous cells can develop in the prostate, which is a gland belonging to the masculine reproductive system. The medical examination leading to a prostate cancer diagnosis is prostate biopsy. It consists in sampling the prostate tissue using a biopsy needle. A proper examination includes twelve samples equally distributed across the prostate volume. Most often, the biopsy needle is positioned and guided by endorectal ultrasound imaging. In 2013, 238.590 new prostate cancer cases have been detected leading to hundreds of thousands biopsy procedures in the USA [1]. Meanwhile, although radical prostatectomy (i.e. surgical ablation of the prostate) remains the most common treatment procedure, novel therapeutic approaches are developed. Among them, brachytherapy, which consists in inserting radioactive seeds

Authors are with UPMC Univ. Paris 06, UMR 7222, ISIR, Paris, France \{poquet, mozer, morel, vitrani\}@isir.upmc.fr.

P.Mozer is also from La Pitié Salpetrière Hospital, Urology Dpt., France This work has been funded by the ANR through the PROSBOT project. with a needle across the prostate volume in order to irradiate the cancerous tissue, is known to generate less side effects than surgery [2], [3].

A major technical difficulty for both prostate biopsy and brachytherapy arises from the required precision for the needle placement. For a biopsy procedure, a precision of a few millimeters for the placement of the biopsy needle tip is desired. In brachytherapy, the desired positioning precision, which is computed in order to optimize the exposure to radiations across the prostate by a dose planning algorithm simultaneously to the seeds activity typically reaches $3 \mathrm{~mm}$. Meanwhile, the prostate has a variable volume, experiences displacements up to $1 \mathrm{~cm}$ or more, and significantly deforms when needles are inserted or when an imaging device such as an ultrasound probe is placed in the rectum in order to monitor the needle placement [4], [5].

It is also important to note that although the technical issues are similar between biopsy and brachytherapy, these two applications face different medico-economic constraints: for a diagnosis biopsy, the duration of the operation as well as the cost of the intervention are more constrained than for a brachytherapy. This probably explains why the literature proposes a wide diversity of approaches and solutions for robotic assistance to needle placement in a patient's prostate. These solutions differ from each other by several technical options for the imagery modality, needle access, robot kinematics and level of automation, see the state of the art analysis detailed in Sec. II.

In Sec. III, we describe the proposed system, which is aimed at assisting prostate biopsies. It is based on the comanipulation concept: a robot holds an endorectal 3D ultrasound probe, which is simultaneously manipulated by the urologist. The registration of the robot with respect to the prostate is made directly from the 3D ultrasound image and using a registration software provided by Koelis (Urostation), [6]. In Sec. IV, the control law proposed for the robot blocking is presented. Finally, in Sec. V, we show experimental results of this system, using both in vitro experiments and validation made in cadavero.

\section{ROBOTIC NEEDLE PLACEMENT IN A PROSTATE}

As mentioned in the introduction, a number of robotic systems for assisting needle positioning in a prostate have been proposed in the literature, see an exhaustive review in [7]. They differ from each other by several technical choices. 


\section{A. Needle access}

Needles can be placed in the prostate either through transperineal access, [7]-[14], or through transrectal access, [15], [16]. For a biopsy procedure, transrectal access is usually preferred. The patient is placed in a lateral decubitus position or in a lithotomy position and a local anesthesia of the rectal region is performed. The patient is awake and may move during the procedure. Endorectal needle placement is associated with endorectal ultrasound imaging (USI): the needle guide is attached to the USI probe. It is important to note that the biopsy needle is not bevelled and has a diameter of approximatively $1 \mathrm{~mm}$, thus its bending is insignificant. The target area of the needle is on the needle guide axis. As a result, the needle position is known in the probe image frame. Prior to its insertion it can be visualized by a straight line on the screen displaying the image.

Transperineal needle placement is generally used for brachytherapy. Here, the patient is placed in the lithotomy position. Some authors also suggest the use of transperineal access for biopsies [17], but the procedure seems longer and the requirement of a total anesthesia seems not compatible with the medico-economic constraints. One advantage of transperineal access is that multiple needles can be inserted simultaneously.

\section{B. Imagery and registration}

Since the prostate deforms and moves with physiological movements and when needles are placed, it is required to monitor the needle placement via real time imaging. Throughout the literature, authors propose to use USI [7], [18], [19], MRI [17], [20], [21] or CT Scan [8].

USI provides either 2D planar images in real time or $3 \mathrm{D}$ images at a few seconds rate. 2D USI is often coupled with a stepper: thanks to successive incremental penetration movements of the probe, a series of parallel cross-sections is acquired and assembled to provide a 3D image [9]-[11], [13], [18], [19]. USI is largely available in urologist consulting rooms at a reasonable cost.

MRI and CT scan imaging provide better images at a higher cost and lower frequency. MRI also imposes drastic constraints on the design of the robot due to magnetic compatibility [17], [20] and CT scan poses problems due to irradiation doses for both the urologist and the patient.

One important issue when coupling a needle placement robotic assistant to an imagery system is the registration of the robot end-effector frame $\mathscr{F}_{e}$, in which the location of the needle frame $\mathscr{F}_{n}$ is known, with the image frame $\mathscr{F}_{i}$, in which the prostate is to be localized by imagery. When using MRI or CT scan, this can be done at the beginning of the procedure by imaging the robot and identifying characteristic points of the robot in the image. From this, the homogeneous transform $\mathbf{T}_{i \rightarrow e}$ between $\mathscr{F}_{i}$ and $\mathscr{F}_{e}$ is known at any time. Thus, when imaging the prostate in $\mathscr{F}_{i}$, it is straightforward to compute the location of the prostate in the robot endeffector frame.

Since the needle guide is fixed with respect to the probe, there is no need to register the robot and the needle when using endorectal USI: $\mathbf{T}_{i \rightarrow n}$ is fixed and known in advance. However, it is still required to register the $\mathscr{F}_{e}$ with the prostate. This can be done by recording a first 3D image $\mathscr{I}_{0}$ of the prostate at an initial position. To do so, one needs to know the location of the probe frame in the robot end effector frame, which is done thanks to standard calibration procedures [22]. From then on, some authors propose to use the robot joint sensors to measure the displacement of the probe with respect to the prostate during operation. However, this may lead to errors because of the prostate movement during the procedure [4]. It seems more precise to use 3D USI since a 3D image can be taken from any position and orientation of the probe. It can thus be register to $\mathscr{I}_{0}$ to estimate the displacement of the probe (thus of the robot and needle) with respect to the prostate. In this case elastic registration is desirable due to the prostate deformation. An iconic registration method has been proposed for fast and robust application in [23], and used as a localizer in a robotic system in [7]. Nowadays this technology is commercially available through a system called Urostation, developed by the French company Koelis. It is approved for clinical practice (CE, FDA).

\section{Robot kinematics}

The number of Degrees of Freedom (DoFs) required to place the tip of a needle at an arbitrary position with an arbitrary needle axis orientation is 5 and not 6 , since the rotation of the needle around its penetration axis does not affect the tip position nor the axis orientation. Some authors use 6 active DoFs, the actuation of the rotation around the needle axis being used to improve the needle penetration through the perineum [7].

When the robot manipulates an endorectal USI probe, the anus plays the role of a 2 DoFs kinematic constraint. Only 4 DoFs are to be used: 3 rotations around the penetration point and 1 translation along the penetration axis. This has led to the design of robots exhibiting a remote center of motion [10]. A clear benefit of this approach is that only 4 actuated degrees of freedom are required, which participates in reducing the cost. A major drawback is that, prior to operation, a setup phase is required to place the remote center of motion which is fixed with respect to the robot base so as to coincide with the patient's anus. Furthermore, urologists can use the human tissue elasticity and lightly press against one side of the rectum to achieve a particular needle placement. This leads to a displacement of the rectum with respect to the prostate that has been observed in [24]. Due to other geometrical constraints (anatomical and from needle guide placement that should grant an easy access to the urologist), it seems to be useful to produce small movements that do not strictly leave the entry point at a fixed position.

\section{Degree of automation}

Systems proposed in the literature also differ in their level of automation. Some devices are fully automated: the robot is registered with respect to the prostate, the needle 
desired location is given by a pre-operative planning and the robot autonomously places the needle [11]. Other devices are comanipulators, in that sense that the gesture control is shared by the robot and the urologist. The most frequent scenario for this approach involves the robot placing the needle guide and the needle itself being placed by the urologist [10], [15], [19].

\section{PROPOSED SYSTEM}

The research presented in this paper is dedicated to the development of a robotic assistant to prostate biopsies. Its main objective is to bring significant improvement in terms of precision and reliability of the needle placement. Secondary objectives include: minimal changes to the current clinical practice, no increase of the biopsy procedure duration, minimal robot installation time and limited additional cost. This has led us to make the following choices:

- An endorectal 3D end-fire ultrasound probe is used for the imagery along with a 3D USI-USI registration algorithm [23].

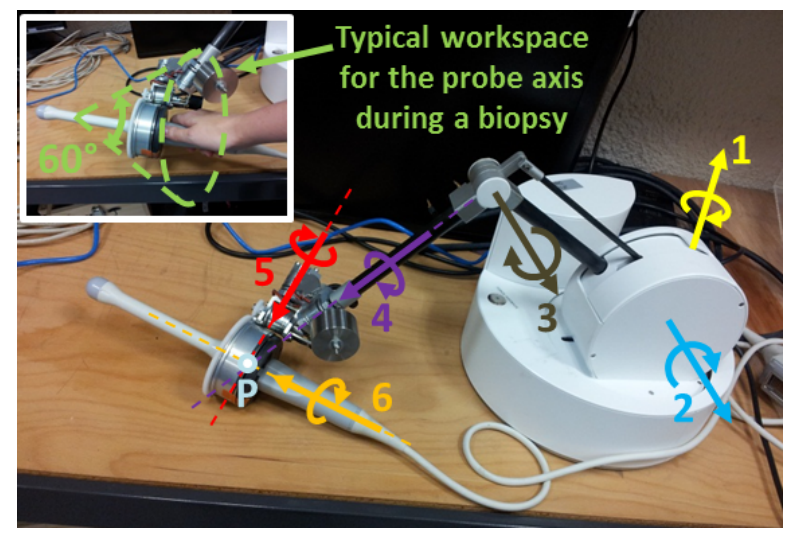

Fig. 1. A general view of the proposed TRUS probe comanipulator

- The comanipulation paradigm is used to position the probe (and therefore, the needle) with respect to the prostate: the robot and the urologist simultaneously manipulate the probe. In this paper, the robot is either blocking the probe motion or letting the urologist freely move the probe. In the scenario, the urologist navigates thanks to 3D or 2D USI and, when he/she has reached the position corresponding to a desired biopsy, he/she blocks the probe. With free hands, the urologist can image the prostate, register the current prostate 3D image with the initial image, visualize the needle position in the prostate and proceed to the biopsy through the needle guide. Note that such a robot could be used for other assistance functions, such as displaying forces in relation to a deformation measured in the ultrasound images in real time, following the principle presented in [25].

- In order to avoid a fastidious setup, where the position of the robot base with respect to the patient's anus is to be finely tuned prior to operation, a 6 DOF kinematics has been preferred to a remote center configuration.
A picture of the comanipulator is given in Fig. 1. In the following, we detail the robot kinematics and its actuation system.

\section{A. Robot kinematics}

As emphasized from clinical data in [24], during a prostate biopsy procedure, the workspace for the probe axis can be modeled by a cone, whose origin coincides with the anatomical entry point, and whose angle is typically 60 degrees, see Fig. 1, upper left corner. The rotation of the probe around its penetration axis is typically more than 300 degrees, as it is used to position the needle guide, whose axis is parallel to the probe penetration axis.

A 6 DoF robot has been designed to be compatible with this workspace, while the robot base is placed on the examination table at a typical distance of $40 \mathrm{~cm}$ from the entry point. It is made of 6 pivot joints serially assembled according to a conventional anthropomorphic geometry: the 3 first pivot joints' axes, namely a vertical axis followed by two parallel horizontal axes, form the shoulder and the elbow while the three last pivot joints' axes, coinciding at a point $P$, form a wrist, see Fig. 1 . The geometry is completely described by Table I according to Denavit and Hartenberg notation, [26], along with the frames $\mathscr{F}_{i}$ attached to each body.

\begin{tabular}{||c|c|c|c|c||}
\hline$i$ & $\alpha_{i-1}$ & $a_{i-1}$ & $d_{i}$ & $\theta_{i}$ \\
\hline 1 & 0 & 0 & 0 & $\theta_{1}$ \\
\hline 2 & $-\pi / 2$ & 0 & 0 & $\theta_{2}$ \\
\hline 3 & 0 & $0.25 \mathrm{~m}$ & 0 & $\theta_{3}$ \\
\hline 4 & $\pi / 2$ & 0 & $0.3 \mathrm{~m}$ & $\theta_{4}$ \\
\hline 5 & $-\pi / 4$ & 0 & 0 & $\theta_{5}$ \\
\hline 6 & $\pi / 2$ & 0 & 0 & $\theta_{6}$ \\
\hline
\end{tabular}

TABLE I

DENAVIT AND HARTENBERG PARAMETERS OF THE COMANIPULATOR

The last pivot axis is designed in such a way that it leaves a $8 \mathrm{~cm}$ diameter cylindrical hole whose axis coincides with the rotational axis. Therefore, an interface part can be designed to adapt to any specific probe shape and to connect to the robot end-effector. This part is fixed on the probe and can be placed into the robot end effector thanks to a mechanical connector involving magnets, see Fig. 2

\section{B. Actuation}

The robot shall be able to apply forces to block the system at an arbitrary position. Conventionally, in robotics, blocking a joint can be done either by an electric motor that is servoed to a fixed position with a high gain (resulting in a high stiffness) or by an electromagnetic brake (which provides an infinite stiffness when not sliding). Electromagnetic brakes present the advantage of a relatively high ratio between the resistive torque and the actuator mass, when compared to an electric motor. They are also significantly cheaper and the control electronics are simpler than for an electric motor. However, they present the drawback of poor controllability: the brake is either on, resulting in an infinite stiffness (as long as the maximum resistive torque is not reach) or off, resulting 


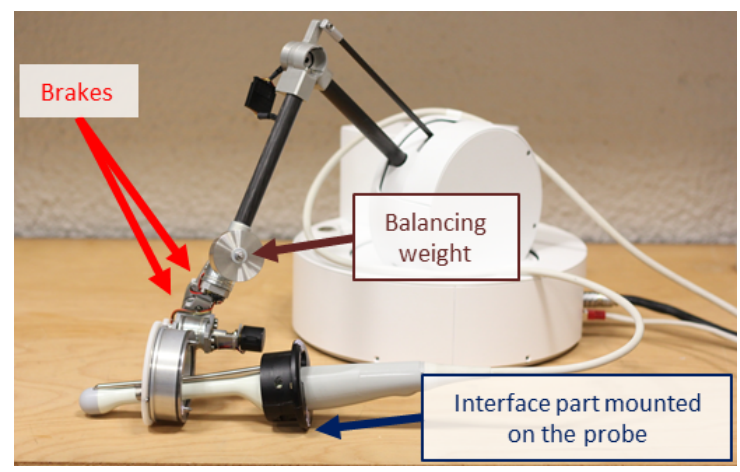

Fig. 2. A view of the interface part used to mount the probe. It allows a 340 degrees rotation of the probe around its axis. A hole is left to insert the biopsy needle guide.

in null stiffness. When designing the actuation system, we had to consider:

- the total mass of the robot, which should be minimized in order to ease the comanipulation in the free mode. This argues for the use of brakes.

- the cost of the device, that should remain low enough in order to make it possible for a transfer to clinical practice. This, again, led us to opt for brakes.

- the requirement for possibly modulating the stiffness to a low value during the blocked phase. Indeed, the patient is awake during the procedure and only benefits from a local anesthesia. Therefore, it is frequent that the patient moves during an examination. This argues for the use of motors.

As a compromise, a hybrid actuation mode was chosen. For the three first robot joints, electric motors are chosen. They will allow to emulate a spring behavior with controllable stiffness for the wrist center point $P$, see Sect. IV. In order to ease the comanipulation in the free mode, resistive forces have to be limited. To this aim:

- the motors are placed near the robot base, in such a way that their mass does not significantly affect the comanipulator inertia.

- a cable transmission is used to limit joint friction.

- load springs are mounted on joints 2 and 3 to compensate for the robot weight.

Each motor (Maxon RE35) is equipped with an optical encoder for position measurement. In the low level electronics, a current loop allows controlling of the motor torque.

For the three last joints, brakes (Kebco 01.P1.300) are used as they provide a solution for applying a high resistive torque with a low mass. Each brake is equipped with a potentiometer to measure the joint position. The robot was manufactured by the French company Haption, [27], and exploits the Haption technology dedicated to high force haptic interfaces for the three first joints.

All the characteristics of the actuation system are summarized in Table II.

\begin{tabular}{||c|c|c|c|c||}
\hline Joint & Actuator & Transmission & Ratio & Max. torque \\
\hline 1 & motor & cable & 21.6 & $3.4 \mathrm{Nm}$ \\
\hline 2 & motor & cable & 14.9 & $2.4 \mathrm{Nm}$ \\
\hline 3 & motor & cable & 14.8 & $2.3 \mathrm{Nm}$ \\
\hline 4 & brake & direct & 1 & $0.4 \mathrm{Nm}$ \\
\hline 5 & brake & direct & 1 & $0.4 \mathrm{Nm}$ \\
\hline 6 & brake & gear & 4.5 & $1.8 \mathrm{Nm}$ \\
\hline
\end{tabular}

TABLE II

ACTUATION DATA

\section{CONTROL}

\section{A. Actuators control input}

The control of the brakes is binary: the brakes are either blocked, which corresponds to the unpowered state or free, which corresponds to powered state. Therefore, in case of a loss of power, the wrist is frozen to its configuration.

The control input for the three first joints' motors is the current $i_{i}, i \in\{1 \cdots 3\}$, which corresponds to a joint torque $\tau_{i}$ up to a scalar factor $k_{\tau_{i}}$ accounting for the motor torque constant and the transmission ratio:

$$
\tau_{i}=k_{\tau_{i}} i_{i}, \quad i \in\{1 \cdots 3\}
$$

In the following, the torque is considered as the control input for the three first joints' motors, knowing that the corresponding input current can be computed thanks to Eq. (1).

\section{B. Force transmission model}

The computation of the torques for the free mode and the blocked mode is primarily based on the kinematic model:

$$
\left(\begin{array}{c}
\mathbf{v}_{6 / 0}(P) \\
\omega_{6 / 0}
\end{array}\right)=\underbrace{\left(\begin{array}{cc}
\mathbf{J}_{v 1} & \mathbf{0} \\
\mathbf{J}_{\omega 1} & \mathbf{J}_{\omega 2}
\end{array}\right)}_{\mathbf{J}} \dot{\mathbf{q}}
$$

where $\mathbf{v}_{a / b}(M)$ stands for the velocity of point $M$ produced in the motion of frame $\mathscr{F}_{a}$ with respect to frame $\mathscr{F}_{b}, \omega_{a / b}$ stands for the rotational velocity of frame $\mathscr{F}_{a}$ with respect to frame $\mathscr{F}_{b}, \dot{\mathbf{q}}=\left[\begin{array}{lll}\dot{\theta}_{1} & \cdots & \dot{\theta}_{6}\end{array}\right]^{\mathrm{T}}$ is the joint velocity vector, $\mathbf{J}$ is the $6 \times 6$ robot jacobian matrix at point $P$ and $\mathbf{J}_{v 1}$, $\mathbf{J}_{\omega 1}$ and $\mathbf{J}_{\omega 2}$ are $3 \times 3$ jacobian sub-matrices. Note that the upper right null sub-matrix indicates that the three last joint movements do not affect the velocity of point $P$.

In the rest of the paper, we will assume full rank for $\mathbf{J}$ (and thus for $\mathbf{J}_{v 1}, \mathbf{J}_{\omega 1}$ and $\mathbf{J}_{\omega 2}$ ), which is practically guaranteed in the prototype due to joint physical limits that leave kinematic singularities out of the workspace.

Due to kinemato-static duality, the transpose of the jacobian matrix defined in Eq. (2) can be used to map an external wrench applied to the environment through the end-effector to the vector of joint torques $\tau=\left[\begin{array}{lll}\tau_{1} & \cdots & \tau_{6}\end{array}\right]^{\mathrm{T}}$ :

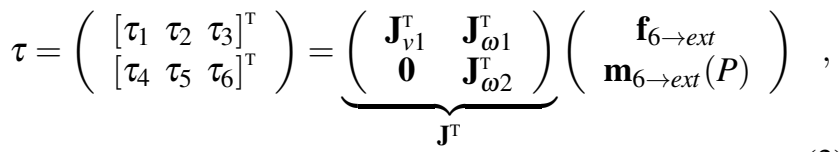


where $\mathbf{f}_{6 \rightarrow \text { ext }}$ is the force applied by the robot end-effector on the environment and $\mathbf{m}_{6 \rightarrow \text { ext }}(P)$ is the moment applied by the robot end-effector on the environment at point $P$.

In the free mode, the brakes being inactive, the joint torques $\tau_{4}$ to $\tau_{6}$ are null. Therefore, from the second line of Eq. (3), it can be seen that the exerted wrench has a null moment at point $P: \mathbf{m}_{6 \rightarrow e x t}(P)=\mathbf{0}$. In other words, Eq. (3) simplifies to:

$$
\left(\begin{array}{lll}
\tau_{1} & \tau_{2} & \tau_{3}
\end{array}\right)^{T}=\mathbf{J}_{v 1}^{\mathrm{T}} \mathbf{f}_{6 \rightarrow e x t} .
$$

In the blocked mode, as long as the brakes are activated and do not slip, bodies 3, 4, 5 and 6 of the robot constitute a same solid body. Both a force and a moment can be applied to the probe at point $P$, but only three actuators are controlled. The mapping from an external wrench applied to the probe to the three active joints torques is obtained by the dual of Eq. (2) when considering that the three last joint velocities are null:

$$
\left(\begin{array}{lll}
\tau_{1} & \tau_{2} & \tau_{3}
\end{array}\right)^{T}=\mathbf{J}_{v 1}^{\mathrm{T}} \mathbf{f}_{6 \rightarrow e x t}+\mathbf{J}_{\omega 1}^{\mathrm{T}} \mathbf{m}_{6 \rightarrow e x t}(P) .
$$

\section{Gravity compensation}

The robot links weight is balanced by counterweights and springs in such a way that there is no need for compensation of the robot weight by the actuators. However, to ease the comanipulation in the free mode and to cancel weight disturbances in the blocked mode, it is desirable to compensate for the weight of the probe and its interface. The external wrench applied to the probe and its interface at point $P$, due to gravity is:

$$
\left(\begin{array}{c}
\mathbf{f}_{g \rightarrow 6} \\
\mathbf{m}_{g \rightarrow 6}(P)
\end{array}\right)=\left(\begin{array}{c}
m \mathbf{g} \\
m \mathbf{d}_{G P} \times \mathbf{g}
\end{array}\right),
$$

where $m$ and $G$ are the mass and the center of gravity of the probe and its interface, $\mathbf{g}$ is the gravitational field vector and $\mathbf{d}_{G P}$ is the vector from $G$ to $P$.

Compensating for gravity straightforwardly derives from Eq. (4) and Eq. (6) in the free mode :

$$
\left(\begin{array}{lll}
\tau_{1} & \tau_{2} & \tau_{3}
\end{array}\right)^{T}=-m \mathbf{J}_{v 1}^{\mathrm{T}} \mathbf{g} .
$$

The resulting behavior, when applying this control law in the free mode, is that weight moments at point $P$ are not compensated for. It is indeed impossible to compensate for weight moments at point $P$ due to freed rotations around point $P$. Assuming that $m$ and $G$ are perfectly known, only gravity is compensated for. In other words, without any other external efforts than gravity, when applying control law Eq. (7), $P$ stays still but the probe orientation around $P$ is free and influenced by gravity.

In the blocked mode, combining Eq. (5) and Eq. (6) leads to the following controller for gravity compensation :

$$
\left(\begin{array}{lll}
\tau_{1} & \tau_{2} & \tau_{3}
\end{array}\right)^{T}=-m\left(\mathbf{J}_{v 1}^{\mathrm{T}} \mathbf{g}+\mathbf{J}_{\omega 1}^{\mathrm{T}}\left(\mathbf{g} \times \mathbf{d}_{G P}\right)\right)
$$

The resulting behavior is that the gravity wrench is balanced by the three motor torques. In other words, the point $P$ and probe orientation stay fixed without any external effort other than gravity.
In the following, gravity is supposed to be perfectly compensated for by the control law (7) in the free mode and by the control law (8) in the blocked mode. For notation simplicity, gravity forces and their compensation are omitted.

\section{Stiffness control for the blocked mode}

To emulate an elastic behavior with a controllable stiffness in the blocked mode, the applied control law is:

$$
\left(\begin{array}{lll}
\tau_{1} & \tau_{2} & \tau_{3}
\end{array}\right)^{T}=\mathbf{J}_{v 1}^{\mathrm{T}}\left(k\left(\mathbf{x}_{\text {ref }}-\mathbf{x}\right)-b \dot{\mathbf{x}}\right),
$$

where $k$ is a stiffness coefficient, $b$ is a viscosity coefficient used for damping out oscillations, $\mathbf{x}$ is the position of point $P$ in the fixed frame $\mathscr{F}_{0}$ and $\mathbf{x}_{\text {ref }}$ is the reference position for $P$. In practice, $\mathbf{x}_{\mathrm{ref}}$ is the end-effector position that is recorded when the urologist activates the blocked mode from the free mode. In other words, it is the position where point $P$ shall remain fixed in the blocked mode.

If an external wrench $\left[\mathbf{f}_{e x t \rightarrow 6}, \mathbf{m}_{e x t \rightarrow 6}(P)\right]$ is applied to the probe, the equilibrium condition obtained by combining Eq. (5) and Eq. (9) for $\dot{\mathbf{x}}=0$ writes:

$$
\mathbf{f}_{e x t \rightarrow 6}+\left(\mathbf{J}_{v 1}^{\mathrm{T}}\right)^{-1} \mathbf{J}_{\omega 1}^{\mathrm{T}} \mathbf{m}_{e x t \rightarrow 6}(P)=k\left(\mathbf{x}_{\text {ref }}-\mathbf{x}\right) .
$$

Therefore, a spring behavior with a stiffness $k$ is obtained at point $P$ when pure forces (i.e. wrenches with a null moment at point $P$ ) are applied. Most likely, both external forces and moments at point $P$ will be applied to the probe by the environment during a biopsy procedure. This will result in a displacement of the probe in position and orientation. Computing this displacement allows to fully determine the 6 DOF stiffness matrix, see the Appendix at the end of the paper.

\section{E. External integration}

In the blocked mode, it is desirable to tune a low stiffness for security reasons. Indeed, during a biopsy procedure, the patient may move and the resulting forces should not be too large. In practice, a stiffness as low as $k=200 \mathrm{~N} / \mathrm{m}$ will be selected. However, in this case, the efforts applied to the rectum and the prostate may induce large displacements.

A typical scenario is the following: the urologist manipulates the probe in the free mode and positions the probe for a biopsy. Doing so, he/she exerts forces on the rectum and the prostate to ensure correct positioning with tight contact between the probe and the rectal wall for proper imaging. An equilibrium is reached between the wrench applied by the urologist and the wrench applied on the patient. The urologist then turns the system into the blocked mode and releases the probe, which should stay still. But since the urologist efforts disappear, only the wrench applied on the patient remains and creates a displacement of the probe according to Eq. (10).

To compensate for this, an outer integral compensation is added. The reference position is changed with a rate:

$$
\dot{\mathbf{x}}_{\text {ref }}=\lambda\left(\mathbf{x}_{\text {refo }}-\mathbf{x}\right),
$$

where $\lambda$ is a scalar gain in $s^{-1}$ and $\mathbf{x}_{\text {ref }}=\mathbf{x}_{\text {ref }}$ when the urologists sets the blocked mode on. In other words:

$$
\mathbf{x}_{\mathrm{ref}}=\mathbf{x}_{\mathrm{ref} 0}+\lambda \int_{0}^{t}\left(\mathbf{x}_{\mathrm{ref} 0}-\mathbf{x}(\tau)\right) d \tau .
$$


Thanks to this integrator, when the urologist releases the probe after switching on the blocked mode, the probe initially moves due to the wrench applied to the patient, but then returns to its desired position thanks to a modification of the reference position.

Combining Eq. (12) with Eq. (9), one finally gets a controller in the blocked mode that could be written as an equivalent conventional PID compensator for the position error, see Fig. 3. The particularity here is the external loop implementation for the integrator and the associated tuning method: a low stiffness $k$ is first chosen; then, potential oscillations are damped out by progressively increasing $b$; finally, the external integral gain $\lambda$ is chosen to tune the time required to compensate for a disturbance. Again, it is not required to select a high value for $\lambda$. A slow compensation will ensure a correction of the position within a few seconds, which is acceptable for the application. It will not significantly change the stiffness at the frequencies that are typical for human-robot interaction (from 0.5 to $3 \mathrm{~Hz}$ ). Furthermore, for safety reasons, the integration can be stopped either when the error becomes null, after a few seconds, or when the force applied by the controller exceeds a tunable limit.

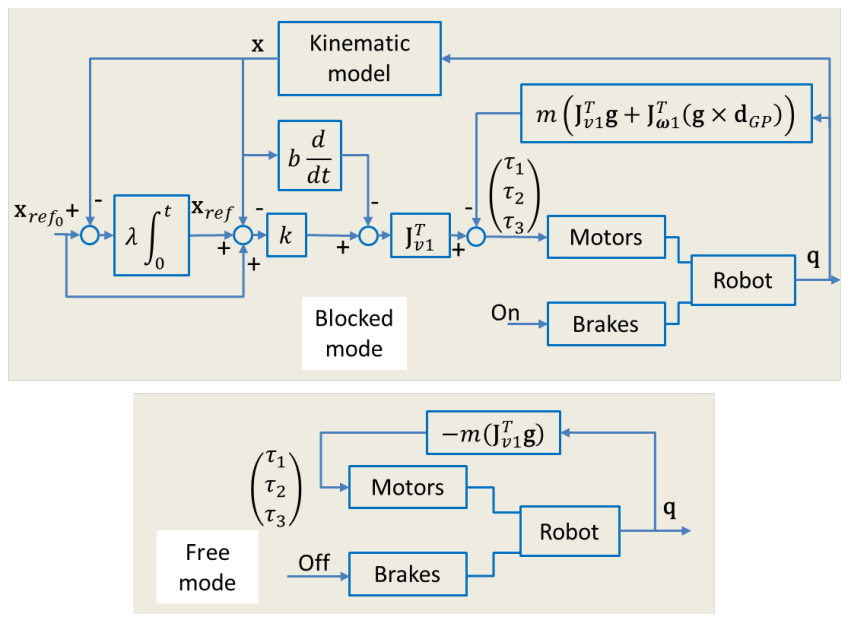

Fig. 3. Scheme of the final controller in the blocked mode (up) and in the free mode(down).

\section{EXPERIMENTAL VALIDATION}

\section{A. Controller tuning}

For proper probe weight compensation, it is first required to estimate parameters $m$ and $\mathbf{d}_{P G}$. Balancing experiments lead to identify $m=0.5 \mathrm{~kg}$ and $\mathbf{d}_{P G}=-d \mathbf{z}_{6}$, where $d=9 \mathrm{~mm}$ and $\mathbf{z}_{6}$ is the unit vector parallel to the probe penetration axis, directed towards the prostate.

The tuning of the controller requires setting 3 scalar parameters. First a low stiffness $(k=200 \mathrm{~N} / \mathrm{m})$ is chosen for security reasons, since a movement by the patient should not generate large forces). With such a low stiffness, it was experimentally found that the residual joint friction of the device, although rather low, was sufficient to damp out the oscillations without using velocity feedback. Therefore, a viscosity $b=0$ was selected. Finally, the external integrator was tuned thanks to experiments in which an error of $1 \mathrm{~cm}$ induced by an external load can be corrected in approximately 5 second thanks to integration. This lead to $\lambda=4 \mathrm{~s}^{-1}$.

\section{$B$. In vitro experiments}

The ability of the proposed system to maintain a probe at a given position was evaluated during in vitro experiments involving comanipulation of the probe inserted in a prostate phantom (model 053, manufactured by CIRS). Figure 4 shows the setup.

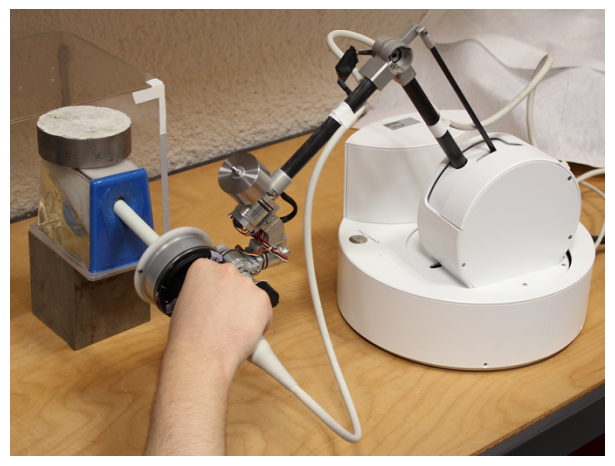

Fig. 4. A picture of the in vitro setup.

The subject is asked to position the probe extremity in contact with the rectal wall toward the prostate while exerting a slight pressure. This corresponds to the clinical configuration, where the urologist pushes the probe in contact with the rectal wall in order to properly image the prostate. When he reaches the desired position, the user sets the blocked mode and then releases the probe. The user is asked to present the probe with five different orientations covering the angular workspace (central, up, down, left, right). Four modes of control are tested, as defined in Table III.

\begin{tabular}{||c|c|c|}
\hline & Integrator OFF & Integrator ON \\
\hline Brakes OFF & Mode 1 & Mode 2 \\
\hline Brakes ON & Mode 3 & Mode 4 \\
\hline
\end{tabular}

TABLE III

DEFINITION OF THE 4 CONTROL MODES

Comparing Modes 1 and 3 with Modes 2 and 4 allows for verifying the effect of the external integrator while comparing Modes 1 and 2 with Modes 3 and 4 allows for verifying whether the brakes should be kept or not for future versions of the comanipulator. Note that in Mode 2 and 4, the integrator is stopped after 10 seconds. The system then stays still with only the low stiffness inner controller running.

Figure 5-left presents the time evolution of the position error of point $P,\left\|\mathbf{x}_{\text {ref } 0}-\mathbf{x}\right\|$, recorded during an example experiment in Mode 1. A large steady state error is observed. It is due to the forces exerted by the prostate phantom on the probe and the low stiffness selected for the robot inner loop due to security constraints. On the other hand, in Fig. 5right, where an example result is presented for Mode 4, it can be seen that the error in the $P$ position converges to 
zero, as expected from the presence of an integrator. The integrator activity can be visualized by the time evolution of $\left\|\mathbf{x}_{\text {ref } 0}-\mathbf{x}_{\text {ref }}\right\|$, which corresponds to the norm of the integrator output.
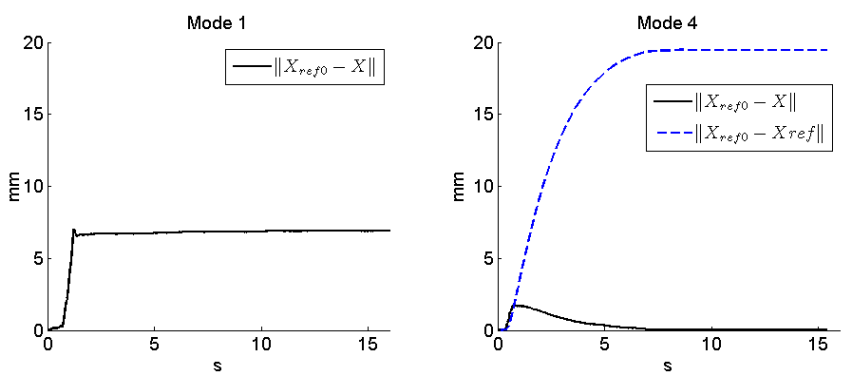

Fig. 5. Evolution of the reference and position along the z-axis during one blockade.

Figure 6 shows the maximal error norm and the steady state error norm for the five experiments in the four modes. As expected, the right column shows that the steady state error is null with the integrator, independently from the fact that the brakes are ON or OFF. The benefit of using brakes lies in the fact that the orientation is maintained constant by the brakes (up to their stiffness, which is high). Therefore, the orientation of $P$ can be kept still as well as its position. Furthermore, when the brakes are OFF, it can be seen in Fig. 6 that the maximal (transient) error is significantly increased at point $P$.
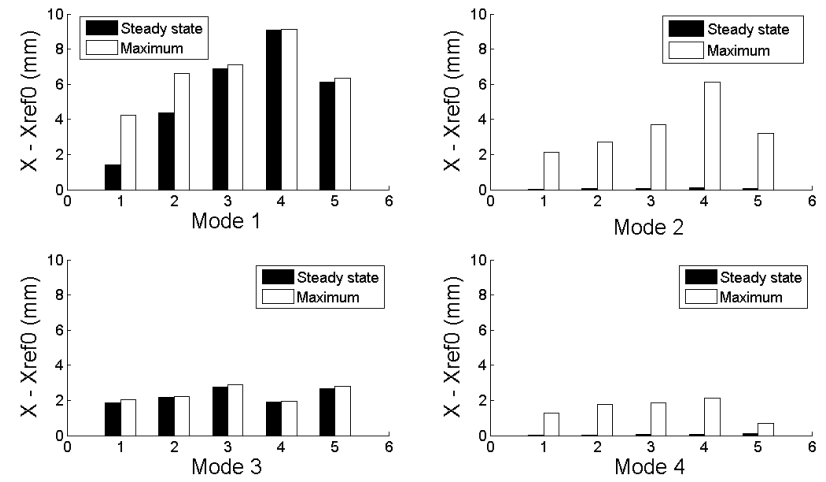

Fig. 6. Maximal and steady state errors for the five experiments in the four modes

\section{In cadavero experiments}

Experiments have been conducted in cadavero at the Surgical School of Assistance Publique - Hopitaux de Paris. Two urologists were comanipulating the system.

A first aim was to verify the geometry. The two urologists were asked to scan the whole prostate with the probe using the ultrasound image, as they would do during a conventional examination. It appeared that the robot workspace was satisfying whether the cadaver was in left lateral decubitus or in lithotomy position, lying down with feet in stirrups. No fastidious setup was required for any of the two body positions: the robot was simply positioned on the table or

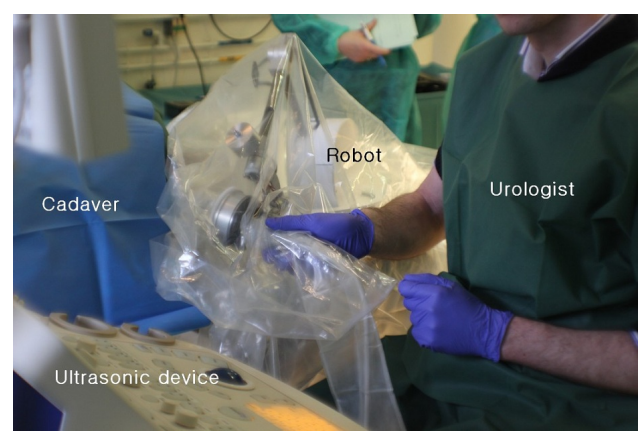

Fig. 7. In cadavero experiments

on a stool, without precise pre-positioning. The first attempt for placing the robot base was satisfactory and convenient to perform all the experiments in both patient positions. Moreover, the urologists declared they felt comfortable and not disturbed in their gesture by the robot in free mode.

Blocking tests were then performed while the probe was inserted in the cadaver rectum and the urologist manipulating the probe was pointing a desired location for a hypothetic biopsy. When the experiments were performed, the controller did not use an external integrator $(\lambda=0$ for these experiments). The stiffness of the inner loop was higher $(k \approx 2000 \mathrm{~N} / \mathrm{m})$. Therefore the steady state positioning error at point $P$ was not null. The average measured probe steady state positioning error for point $P$ was $0.6 \mathrm{~mm}$ across 20 blocking experiments in different configurations.

During the blocking experiments, the change of position of the probe was also measured thanks to 3D USI provided by the probe: before blocking, the urologist acquired a first 3D image. Then, after blocking, another 3D image was acquired. Iconic registration of the two 3D images was then performed thanks to the algorithm provided in [23]. It was found that the average displacement was $3.1 \mathrm{~mm}$ and 3.4 degrees. These results indicate that the orientation changes, in such a way that the probe extremity moves more than point $P$. This is interpreted as a consequence of the non-infinite stiffness of the brakes and the wrist structure. Nevertheless, the precision obtained during this experiments is compatible with the precision required for the gesture.

\section{CONCLUSION}

In this paper, we presented the design of a comanipulator for assisting endorectal prostate biopsies. This lightweight system based on conventional robotic components possesses 6 degrees of freedom but uses only 3 electric motors and 3 basic brakes. It features a free mode, where its low friction and inertia allows for natural manipulation of the probe, and a blocked mode, exhibiting both a very low stiffness and a high steady state precision. It can be registered to the prostate by the use of 3D-3D USI registration algorithms.

A step toward clinical application was made thanks to in cadavero experiments, as the robot appeared to bring significant help in the blocked mode while not disturbing the urologist in the free mode. Current developments include a quantified characterization of the ease of manipulation 
in the free mode and a quantification of the brakes and wrist stiffness. Indeed, in cadavero experiments, coupled with ultrasound imaging of the prostate, emphasized that the orientation is not kept exactly constant by the system. One future development will deal with expressing the control reference in the ultrasound image frame (visual servoing), in order to improve the effective precision.

\section{APPENDIX}

In order to compute the $6 \mathrm{DoF}$ stiffness matrix arising from the control law Eq. (9), let's recall that when an external wrench $\left[\mathbf{f}_{e x t \rightarrow 6}, \mathbf{m}_{\text {ext } \rightarrow 6}(P)\right]$ is applied to the probe, the equilibrium condition is written as:

$$
\mathbf{f}_{e x t \rightarrow 6}+\left(\mathbf{J}_{v 1}^{\mathrm{T}}\right)^{-1} \mathbf{J}_{\omega 1}^{\mathrm{T}} \mathbf{m}_{\text {ext } \rightarrow 6}(P)=k\left(\mathbf{x}_{\mathrm{ref}}-\mathbf{x}\right) .
$$

Assuming an infinitesimal displacement $\left(\mathbf{x}_{\mathrm{ref}}-\mathbf{x}\right)=\delta_{P}$ for point $P$, one gets a joint displacement $\delta_{\mathbf{q}}$ of the three first joints (the three last joints staying still due to the brakes):

$$
\delta_{\mathbf{q}}=\left(\mathbf{J}_{v 1}\right)^{-1} \delta_{P}
$$

which corresponds to an infinitesimal rotation:

$$
\delta_{\mathbf{r}}=\mathbf{J}_{\omega 1} \delta_{\mathbf{q}}=\mathbf{J}_{\omega 1}\left(\mathbf{J}_{v 1}\right)^{-1} \delta_{P},
$$

Combining this three last equations leads to:

$$
\left(\begin{array}{cc}
\mathbf{I} & \left(\mathbf{J}_{v 1}^{\mathrm{T}}\right)^{-1} \mathbf{J}_{\omega 1}^{\mathrm{T}} \\
\mathbf{J}_{\omega 1} \mathbf{J}_{v 1}^{-1} & \mathbf{J}_{\omega 1}\left(\mathbf{J}_{v 1}^{\mathrm{T}} \mathbf{J}_{v 1}\right)^{-1} \mathbf{J}_{\omega 1}^{\mathrm{T}}
\end{array}\right)\left(\begin{array}{c}
\mathbf{f}_{\text {ext } \rightarrow 6} \\
\mathbf{m}_{\text {ext } \rightarrow 6}(P)
\end{array}\right)=k\left(\begin{array}{c}
\delta_{P} \\
\delta_{\mathbf{r}}
\end{array}\right),
$$

which defines the $6 \mathrm{DoF}$ stiffness tunable by the scalar $k$.

\section{REFERENCES}

[1] "American cancer society." [Online]. Available: http://www.cancer.org

[2] P. Stattin, E. Holmberg, J.-E. Johansson, L. Holmberg, J. Adolfsson, J. Hugosson, and on behalf of the National Prostate Cancer Register (NPCR) of Sweden, "Outcomes in localized prostate cancer: National prostate cancer register of sweden follow-up study," Journal of the National Cancer Institute, vol. 102, no. 13, pp. 950-958, 2010.

[3] G. Lu-Yao, P. Albertsen, D. Moore, and et al., "Outcomes of localized prostate cancer following conservative management," JAMA, vol. 302, no. 11, pp. 1202-1209, 2009.

[4] N. N. Stone, J. Roy, S. Hong, Y.-C. Lo, and R. G. Stock, "Prostate gland motion and deformation caused by needle placement during brachytherapy," Brachytherapy, vol. 1, no. 3, pp. 154 - 160, 2002.

[5] V. Lagerburg, M. A. Moerland, J. J. Lagendijk, and J. J. Battermann, "Measurement of prostate rotation during insertion of needles for brachytherapy," Radiotherapy and Oncology, vol. 77, no. 3, pp. 318 $-323,2005$.

[6] "Koelis." [Online]. Available: http://www.koelis.fr

[7] N. Hungr, M. Baumann, J. Long, and J. Troccaz, "A 3-d ultrasound robotic prostate brachytherapy system with prostate motion tracking," IEEE Transactions on Robotics, vol. 28, no. 6, pp. 1382-1397, Dec. 2012.

[8] G. Fichtinger, T. L. DeWeese, A. Patriciu, A. Tanacs, D. Mazilu, J. H. Anderson, K. Masamune, R. H. Taylor, and D. Stoianovici, "System for robotically assisted prostate biopsy and therapy with intraoperative ct guidance," Academic Radiology, vol. 9, no. 1, pp. 60 - 74, 2002.

[9] L. Phee, D. Xiao, J. Yuen, C. F. Chan, H. Ho, H. Choon, C. Christopher, and S. N. Wan, "Ultrasound guided robotic system for transperineal biopsy of the prostate," in Robotics and Automation, 2005. ICRA 2005. Proceedings of the 2005 IEEE International Conference on, April, pp. 1315-1320.

[10] Z. Wei, M. Ding, D. Downey, and A. Fenster, "3d trus guided robot assisted prostate brachytherapy," in Medical Image Computing and Computer-Assisted Intervention MICCAI 2005, ser. Lecture Notes in Computer Science, J. Duncan and G. Gerig, Eds. Springer Berlin Heidelberg, 2005, vol. 3750, pp. 17-24.
[11] H. Bassan, T. Hayes, R. Patel, and M. Moallem, "A novel manipulator for 3d ultrasound guided percutaneous needle insertion," in Robotics and Automation, 2007 IEEE International Conference on, April 2007, pp. 617-622.

[12] T. Podder, W. S. Ng, and Y. Yu, "Multi-channel robotic system for prostate brachytherapy," in Engineering in Medicine and Biology Society, 2007. EMBS 2007. 29th Annual International Conference of the IEEE, Aug.2007, pp. 1233-1236.

[13] G. Fichtinger, J. P. Fiene, C. W. Kennedy, G. Kronreif, I. Iordachita, D. Y. Song, E. C. Burdette, and P. Kazanzides, "Robotic assistance for ultrasound-guided prostate brachytherapy," Medical Image Analysis, vol. 12 , no. 5 , pp. $535-545,2008$, special issue on the 10th international conference on medical imaging and computer assisted intervention - MICCAI 2007.

[14] M. R. van den Bosch, M. R. Moman, M. van Vulpen, J. J. Battermann, E. Duiveman, L. J. van Schelven, H. de Leeuw, J. J. W. Lagendijk, and M. A. Moerland, "Mri-guided robotic system for transperineal prostate interventions: proof of principle," Physics in Medicine and Biology, vol. 55, no. 5, p. N133, 2010.

[15] C. Schneider, A. Okamura, and G. Fichtinger, "A robotic system for transrectal needle insertion into the prostate with integrated ultrasound," in Robotics and Automation, 2004. Proceedings. ICRA '04. 2004 IEEE International Conference on, vol. 1, April-1 May, pp. 365370 Vol.1.

[16] A. Krieger, I. Iordachita, P. Guion, A. Singh, A. Kaushal, C. Menard, P. Pinto, K. Camphausen, G. Fichtinger, and L. Whitcomb, "An mricompatible robotic system with hybrid tracking for mri-guided prostate intervention," Biomedical Engineering, IEEE Transactions on, vol. 58, no. 11, pp. 3049-3060, Nov. 2011.

[17] S.-E. Song, N. Cho, G. Fischer, N. Hata, C. Tempany, G. Fichtinger, and I. Iordachita, "Development of a pneumatic robot for mri-guided transperineal prostate biopsy and brachytherapy: New approaches," in Robotics and Automation (ICRA), 2010 IEEE International Conference on, May 2010, pp. 2580-2585.

[18] S. Salcudean, T. Prananta, W. Morris, and I. Spadinger, "A robotic needle guide for prostate brachytherapy," in Robotics and Automation, 2008. ICRA 2008. IEEE International Conference on, May 2008, pp. 2975-2981.

[19] H. Ho, P. Mohan, E. Lim, D. Li, J. Yuen, W. Ng, W. Lau, and C. Cheng, "Robotic ultrasound-guided prostate intervention device: system description and results from phantom studies," The International Journal of Medical Robotics and Computer Assisted Surgery, vol. 5, no. 1, pp. 51-58, 2009.

[20] G. Fischer, I. Iordachita, C. Csoma, J. Tokuda, S. DiMaio, C. Tempany, N. Hata, and G. Fichtinger, "Mri-compatible pneumatic robot for transperineal prostate needle placement," Mechatronics, IEEE/ASME Transactions on, vol. 13, no. 3, pp. 295-305, June 2008.

[21] A. Patriciu, D. Petrisor, M. Muntener, D. Mazilu, M. Schar, and D. Stoianovici, "Automatic brachytherapy seed placement under mri guidance," Biomedical Engineering, IEEE Transactions on, vol. 54, no. 8, pp. 1499-1506, Aug.2007.

[22] M.-A. Vitrani and G. Morel, "Hand-eye self-calibration of an ultrasound image-based robotic system," in Intelligent Robots and Systems, 2008. IROS 2008. IEEE/RSJ International Conference on, Sept.2008, pp. 1179-1185.

[23] M. Baumann, P. Mozer, V. Daanen, and J. Troccaz, "Prostate biopsy tracking with deformation estimation," Medical Image Analysis, vol. 16, no. 3, pp. $562-576,2012$.

[24] C. Torterotot, P. Mozer, M. Baumann, M. Vitrani, and G. Morel, "Analysis of endorectal probe kinematics during prostate biopsies," in Proc. of the Hamlyn Symposium on Medical Robotics, ISBN: 9780-9563776-1-6, 2010, pp. 47-48.

[25] C. Torterotot-Poquet, M.-A. Vitrani, P. Mozer, and G. Morel, "Ultrasound image-based comanipulation for enhanced perception of the contacts with a distal soft organ," in Robotics and Biomimetics (ROBIO), 2011 IEEE International Conference on, Dec.2011, pp. 1140-1146.

[26] J. Denavit and R. S. Hartenberg, "A kinematic notation for lower-pair mechanisms based on matrices," Trans ASME J. Appl. Mech, vol. 23, pp. 215-221, 1955.

[27] "Haption." [Online]. Available: http://www.haption.fr 